\title{
Optical vortex production mediated by azimuthal index of radial polarization
}

\author{
Ignacio Lopez-Quintas ${ }^{1}$, Warein Holgado ${ }^{1}$, Rokas Drevinskas ${ }^{2}$, Peter G. Kazansky ${ }^{2}$, \\ íñigo J. Sola ${ }^{1}$ and Benjamín Alonso ${ }^{1 *}$ \\ ${ }^{1}$ Grupo de Aplicaciones del Láser y Fotónica (ALF), Departamento de Física Aplicada, University of Salamanca, E37008 Salamanca, \\ Spain \\ ${ }^{2}$ Optoelectronics Research Centre, University of Southampton, SO17 1BJ Southampton, United Kingdom
}

E-mail: * $\underline{\text { b.alonso@usal.es }}$

\begin{abstract}
Special light beams are becoming more and more interesting due to their applications in particle manipulation, micromachining, telecommunications or light matter-interaction. Both spin and orbital angular momenta of light are exploited often in combination with spatially varying linear polarization profiles (e.g. radial or azimuthal distributions). In this work we study the interaction between those polarization profiles and the spin-orbit angular momenta, finding the relation involved in the mode coupling. We find that this manipulation can be used for in-line production of collinear optical vortices with different topological charges, which can be filtered or combined with controlled linear polarization. The results are valid for continuous wave and ultrashort pulses, as well as for collimated and focused beams. We theoretically demonstrate the proposal, which is further confirmed with numerical simulations and experimental measurements with ultrashort laser pulses.
\end{abstract}

Keywords: vortex beams, ultrashort pulses, orbital angular momentum

\section{Introduction}

During the last few years the applications of structured light with spatial polarization shaping and orbital angular momentum (OAM) are becoming increasingly important in many fields [1,2] as light-matter interaction [3], micromachining [4], particle acceleration, microscopy [5] or telecomunications, among others. The spin angular momentum (SAM) of light also plays an important role in those applications, e.g. to control polarization and OAM in attosecond pulses [6].

The coupling between spatial phase in OAM and circular polarization in SAM has been known for a long time [7], which remains being a topic under investigation. In this way, spin-orbit interaction has previously been reported to especially play an important role in nano-optics, photonics and plasmonics [8], being applied for example to chiral nanophotonic waveguides [9]. In previous works it has been studied in the far-field at sub-wavelength scale [10]. Recently, electromagnetically induced transparency has been used to couple spin-orbit [11].

Spatially-varying polarization extensively used as radial or azimuthal polarization can be imparted, for example, by liquid crystals [12] and spatial light modulators [13] that can be combined with interferometry [14], or with nanostructured inscribed gratings waveplates $[15,16]$. A polarization azimuthal index, $m$, can be associated to these polarization patterns, being $m$ defined as the number of times that the orientation of the local linear polarization distribution is rotating $2 \pi$ radians for a variation of $2 \pi$ radians in the azimuthal coordinate (both usual radial and azimuthal polarization possess $m=1$ ).

Radial and azimuthal polarizations can interact with the OAM of the light. In previous works, it has been reported the interaction of polarization azimuthal index with OAM under tight focusing conditions [17], the radial to azimuthal polarization conversion using high-order vortices and tight focusing [18], decomposition of linearly polarized vector beams without vortex structure into two orthogonal vortex 
fields, each one exhibiting OAM with opposite charge $m$ [19], the production of spatially separated OAM modes by geometry, polarization and nonlinear process control [20], the use of polarization azimuthal index to detect OAM under tight focusing [21], and transformation of perturbated polarization singularities into topological charges [22]. Also, diffractive optical elements have been applied to radially polarized beams to spatially separate circularly polarized vortices with opposite helicity [23] and, very recently, it has been measured the spatial phase of vector beams decomposed in vortex beams with circular polarization [24]. Interferometric techniques have also been reported for OAM modes transformation [25]. Finally, the advantages of ultrashort laser pulses and particular polarization patterns combined with OAM have been exploited in several applications [26-30].

In this work, we propose the use of a laser beam with azimuthally varying linear polarization to create collinear optical vortices with different OAM. Depending on the polarization azimuthal index of the polarization pattern, there is a different transfer of OAM to the final vortices, which is added to the possible input OAM. We first demonstrate the proposal and study the angular momentum -both OAM and SAM- properties of the decomposed beams, verifying the momentum conservation, and showing that the SAM of the vortices can be transferred.

After analytically demonstrating the angular momentum transference, we numerically show the vortices production and simulate their focusing. Then, we perform experiments with a femtosecond pulsed laser, demonstrating the OAM of the final vortices with different input beams. Notice that the proposed method is not specific for ultrashort pulses and can be applied either to pulsed or continuous wave radiation.

\section{Methods}

\subsection{Theoretical demonstration}

We consider a beam with a transverse distribution of the polarization, $R_{m}^{\ell_{0}}$, denoting the polarization azimuthal index by $m$ and the input OAM by $\ell_{0}$. The beam is defined as

$$
R_{m}^{\ell_{0}}=e^{i \ell_{0} \phi}\left(\begin{array}{c}
\cos (m \phi) \\
\sin (m \phi)
\end{array}\right),
$$

where $\phi$ denotes the azimuthal coordinate. The beam presents a singularity at the center of coordinates both due to the spatiallyvarying polarization and to the OAM introduced by the s-waveplate, being a zero of intensity at posterior propagation positions. As the radially polarized beam is locally linearly polarized and the SAM of linear polarization is $\sigma=0$, thus it does not carry any global SAM, so that the total angular momentum, $j$, of the input beam, $R_{m}^{\ell_{0}}$, is $j_{0}=\ell_{0}+\sigma_{0}=\ell_{0}$ (note that the input beam energy is normalized).

We define the normalized right-hand circular polarization (RCP) and left-hand circular polarization (LCP) states as

$$
\begin{aligned}
& R C P=\frac{1}{\sqrt{2}}\left(\begin{array}{c}
1 \\
-i
\end{array}\right) \\
& L C P=\frac{1}{\sqrt{2}}\left(\begin{array}{c}
1 \\
+i
\end{array}\right)
\end{aligned}
$$

where the SAM of the RCP and LCP beams are defined to be $\sigma_{R C P}=-1$ and $\sigma_{L C P}=+1$, respectively.

Then, the spatially-varying polarization of the vector beam can be re-expressed as

$$
\left(\begin{array}{c}
\cos (m \phi) \\
\sin (m \phi)
\end{array}\right)=\frac{e^{+i m \phi}}{2}\left(\begin{array}{c}
1 \\
-i
\end{array}\right)+\frac{e^{-i m \phi}}{2}\left(\begin{array}{c}
1 \\
+i
\end{array}\right)
$$

which represents the superposition with equal amplitude of two circularly polarized beams of opposite handedness, RCP and LCP, which carry opposite OAM equal to $\ell=+m$ and $\ell=-m$, respectively.

Considering it, the original radially polarized beam, $R_{m}^{\ell_{0}}$, can be expressed as

$$
R_{m}^{\ell_{0}}=\frac{e^{i\left(\ell_{0}+m\right) \phi}}{\sqrt{2}} R C P+\frac{e^{i\left(\ell_{0}-m\right) \phi}}{\sqrt{2}} L C P
$$

which means the superposition with equal amplitude of two beams: the first with OAM value given by $\ell_{1}=\ell_{0}+m$ and with RCP, therefore with SAM $\sigma_{1}=-1$; while the second beam having $\ell_{2}=\ell_{0}-m$ and with LCP, thus with SAM $\sigma_{2}=+1$.

The total angular momenta of the decomposed beams are

$$
\begin{aligned}
& j_{1}=\ell_{1}+\sigma_{1}=\ell_{0}+m-1 \\
& j_{2}=\ell_{2}+\sigma_{2}=\ell_{0}-m+1
\end{aligned}
$$

and it is found that the angular momentum is conserved: $j_{\text {total }}=\left(j_{1}+j_{2}\right) / 2=\ell_{0}$ (equal to the original beam $R_{m}^{\ell_{0}}$ ), taking into account the energy of each beam.

If we apply a quarter-waveplate (QWP) oriented at $45^{\circ}$, denoted by the matrix $Q$, the RCP and LCP are converted to horizontal and vertical linear polarization, respectively:

$$
Q \cdot R_{m}^{\ell_{0}} \propto \frac{e^{i\left(\ell_{0}+m\right) \phi}}{2}\left(\begin{array}{l}
1 \\
0
\end{array}\right)+\frac{e^{i\left(\ell_{0}-m\right) \phi}}{2}\left(\begin{array}{l}
0 \\
1
\end{array}\right)
$$


Due to the QWP, the SAM of both components is eliminated, $\sigma_{1}=-1 \rightarrow 0$ and $\sigma_{2}=+1 \rightarrow 0$, respectively, and there is not a global transfer of SAM to the light beam. The OAM of the decomposed beams is not altered at this stage, therefore producing two orthogonal linearly polarized optical vortices with relative OAM given by $\Delta \ell=\ell_{1}-\ell_{2}=2 \mathrm{~m}$. Both vortices are produced with the same energy and ideally without energy loses. Because of having perpendicular polarization, the vortices can be independently filtered just by means of a linear polarizer or they can be projected to the same linear polarization with the desired relative weight in order to be used for a certain application.

Finally, the focusing of the different vortices was numerically simulated by calculating the electric field following the Fresnel's diffraction model as described in $[31,32]$. The model approximation is valid close to the optical axis, which is appropriate for focused beams.

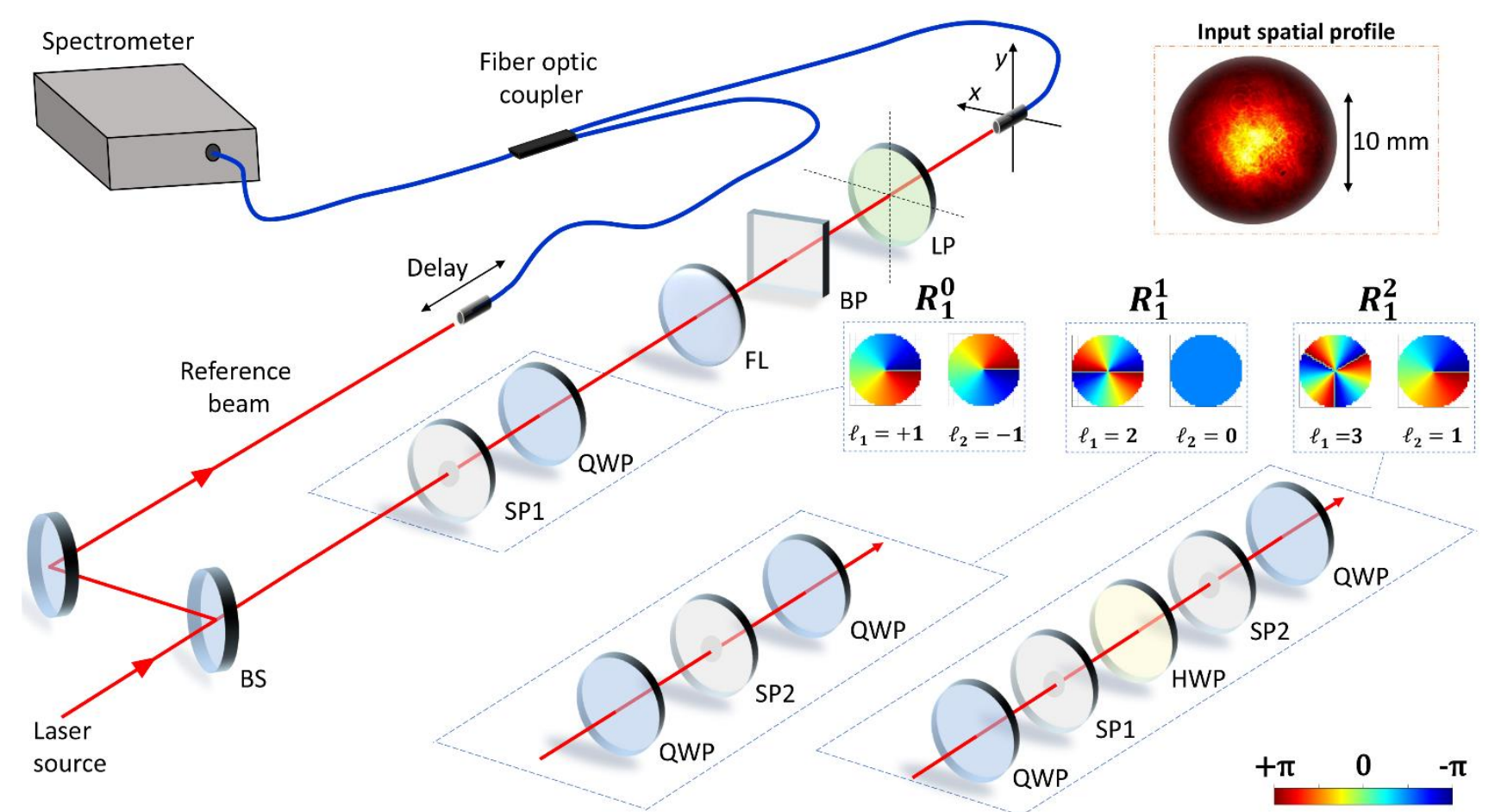

Figure 1. Schematic representation of the three different experimental configurations used for the generation and characterization of the vortex beams with radial polarization modes $R_{m}^{\ell_{0}}$, where $\ell_{0}$ and $m$ denote the input OAM and the azimuthal index respectively. The insets show the spatial phase (wavefront) of the decomposed orthogonal projections with their corresponding OAM $\ell_{1}$ and $\ell_{2}$ for each studied vortex beam. Characterization was performed using the STARFISH technique and in-line interferometry. Labels in the figure correspond to: BS, beam splitter; SP, S-plate (SP1 was oriented to produce radial polarization for input horizontal linear polarization, while the orientation for SP2 was arbitrary); QWP, quarterwaveplate $\left(45^{\circ}\right)$; HWP, half-waveplate (arbitrary orientation); FL, focusing lens; BP, birefringent plate and LP, linear polarizer (set at $0^{\circ}, 90^{\circ}$ and $45^{\circ}$ for analysing the different projections). For STARFISH the fibre optic coupler is used to combine the delayed reference pulse and the beam under test. The spatial profile of the test beam is scanned using an $x, y$ motorized stage (not shown) after passing through the in-line interferometry section of the set-up, composed by the BP and the LP. The FL was present only for the focusing measurements. Inset: the spatial profile of the input beam is shown.

\subsection{Experimental set-up}

A schematic representation of the three different configurations of the experimental set-up employed in this work is depicted in figure 1 . For the experiments, we used as a source Ti:sapphire CPA laser system delivering linearly polarized pulses centered at $797 \mathrm{~nm}$ with a Fourier limit of
$80 \mathrm{fs}$ (FWHM). The radially polarized beam with input OAM was generated by using two different S-plates (SPs) $[15,16]$. The first of them, SP1, operates as half-waveplate with spatially varying fast axis, and converts input linear polarization into radial polarization $(m=1)$ or input circular polarization into circular polarization vortex $(\ell= \pm 1$, depending on input helicity sign) [15]. The second plate, SP2, operates as quarter-waveplate with spatially varying fast axis, 
and converts input circular polarization into radial polarization $(m=1)$ vortex $(\ell= \pm 1)$ [16]. We used two zero-order QWPs designed for $800 \mathrm{~nm}$, the first to produce the input circular polarization and the other to decompose the perpendicularly linear polarized vortices from the radially polarized beams. After this decomposition, the beams where focused by an achromatic lens (focal length $f=500 \mathrm{~mm}$ ).

In both cases, collimated and focused beam, the spatiotemporal (and spatiospectral) amplitude and phase of the $\mathrm{X}$ and $\mathrm{Y}$ polarization components were measured with the STARFISH technique [33], while their relative phase were measured by means of spatially-resolved in-line interferometry [34], taking advantage of the recently introduced capability for ultrafast vector beams reconstruction [35]. Therefore, the experimental method is able to extract the spatiotemporal polarization as well as the relative wavefronts between the $\mathrm{X}$ and $\mathrm{Y}$ projections.

\section{Results and discussion}

\subsection{Simulation of vortex beams}

We firstly performed the numerical simulations of a collimated pulsed laser beam having a gaussian spatial transverse profile of 4-mm diameter (FWHM). The input pulses had a duration of 80 fs (intensity FWHM). Then, we calculated different radially polarized vortices, $R_{m}^{\ell_{0}}$, and applied the decomposition in output modes described above, producing linearly polarized vortices with different OAM, $\ell_{1}$ and $\ell_{2}$.

As an example, we show the spatial phase (figure 2) of the $\mathrm{X}$ and Y polarization components for input vector beam $R_{1}^{1}$, producing modes with $\ell=2$ and $\ell=0 ; R_{1}^{2}$, producing modes $\ell=3$ and $\ell=1 ; R_{2}^{0}$, producing modes $\ell=2$ and $\ell=-2$; and $R_{2}^{2}$, producing modes $\ell=4$ and $\ell=0$. The predicted OAMs are checked in the wavefronts (figure 2), and the relation $\Delta \ell=2 m$ is also verified. As expected, the results are constant throughout the spectral and temporal ranges.

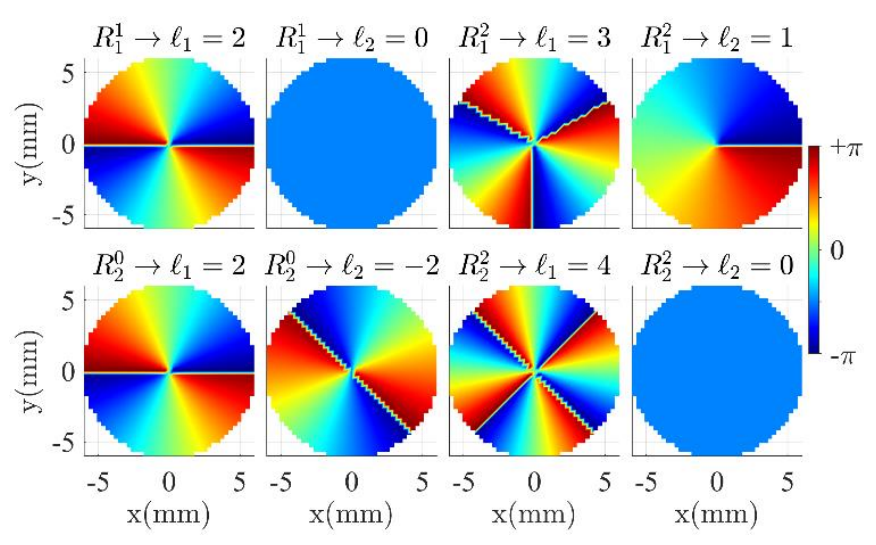

Figure 2. Simulation of collimated radially polarized vortices, $R_{m}^{\ell_{0}}$, projected to orthogonally linearly polarized vortices. The spatial phase (wavefront) of different output OAM, $\ell_{1}$ and $\ell_{2}$, is represented.

Then, we performed numerical simulations under loose focusing of the produced ultrashort beams. Focusing was simulated using a lens with focal length $f=500 \mathrm{~mm}$, as in the experiments, being the results of the spatial intensity and phase at focus presented in figure 3 for each considered input beam.

For example, when the input of the simulation corresponds to vector beam $R_{1}^{2}$, the OAM of the two vortices produced are 3 and 1 respectively, which is deduced from the $\mathrm{X}$ and $\mathrm{Y}$ wavefronts and in the comparison between the ring diameter of the focusing vortices (figure 3 , row 1 ).

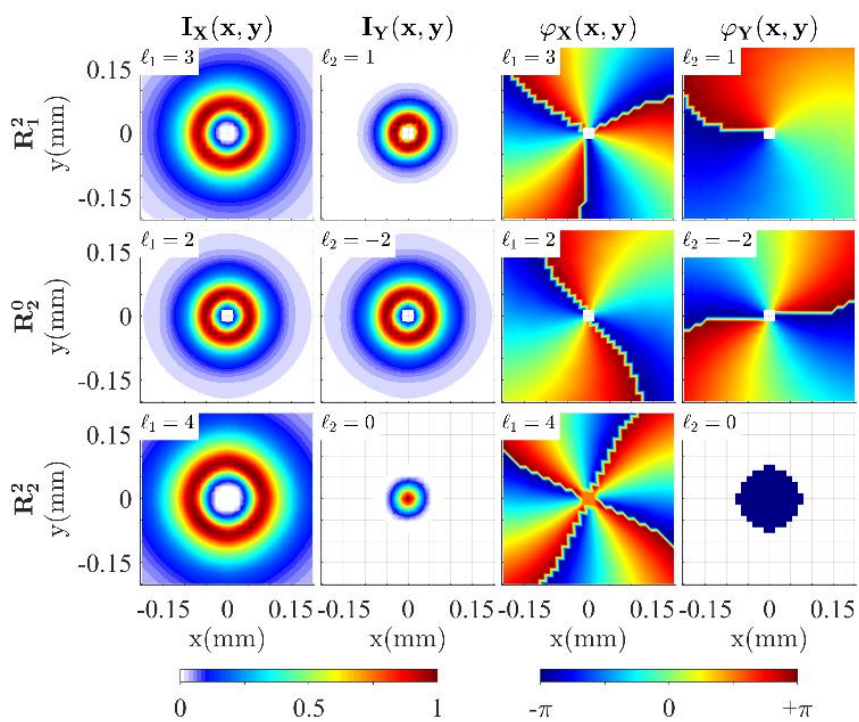

Figure 3. Focusing different vortices produced from $R_{m}^{\ell_{0}}$ modes. $\mathrm{X}$ and $\mathrm{Y}$ components of the spatial intensity (columns 1 and 2) and phase (columns 3 and 4). Rows 1, 2 and 3, starting from the top, correspond to input $R_{1}^{2}, R_{2}^{0}$ and $R_{2}^{2}$, 
respectively. Notice that the intensity plots are individually normalized.

Input $R_{2}^{0}$ produces modes with OAM 2 and -2 , therefore having the same spatial intensity ring, while having opposite variation of the spatial phase (figure 3 , row 3 ). In the case of input $R_{2}^{2}$, the decomposed vortices have topological charges of 4 and 0 , the first of them having larger ring diameter compared to lower values of OAM, while the second one does not feature singularity (figure 3 , row 3 ). Here, the results are also invariant with time and frequency.

\subsection{Experimental vortex production}

With the elements described in the Methods section, we were able to generate three different modes $R_{m}^{\ell_{0}}$ (disregarding the equivalents just with different signs). Using input linear polarization, we produced $R_{1}^{0}$ with SP1. Illuminating with circular polarization produces $R_{1}^{1}$ when using SP2, and $R_{1}^{2}$ when using SP1 followed by SP2.

In this latter case $\left(R_{1}^{2}\right)$ we used an achromatic half-wave plate to change the helicity sign of the circular polarization in between (see figure 1), otherwise the OAM introduced by SP2 would result in cancelling the OAM after SP1, therefore producing $R_{1}^{0}$ instead of $R_{1}^{2}$.

Firstly, we experimentally generated those modes in a collimated configuration, and then we measured their wavefront. If a tilt in the spatial scan of the measured beam with STARFISH is deliberately introduced, the result is equivalent to the measurement of the interference of the beam with a plane wave. Therefore, by this means, we obtained a measurement of the OAM present in the different configurations. In figure 4 we show the interference fringes when producing vortices from radial modes $R_{1}^{2}$ and $R_{1}^{1}$.

The spatial measurement was performed in the central region of the beam, with a two-dimensional scan in a square grid of 1-mm using a step of $40 \mu \mathrm{m}$. In the first case, $R_{1}^{2}$, the $\mathrm{X}$ and $\mathrm{Y}$ components of the decomposed vortices correspond to $\ell_{1}=3$ (figure $4 \mathrm{a}$ ) and $\ell_{2}=1$ (figure $4 \mathrm{~b}$ ), respectively.

In the case $R_{1}^{1}$, the $\mathrm{X}$ and $\mathrm{Y}$ components correspond to $\ell_{1}=2$ (figure $4 \mathrm{c}$ ) and $\ell_{2}=0$ (figure $4 \mathrm{~d}$ ). The experimental results confirm the generation of the predicted OAMs. The small noise in the fringes is due to interferometric instability in the detection because of scanning the beam in the $25 \times 25$ points grid.

For $\ell_{1}=3$ (figure $4 \mathrm{a}$ ), it is seen that the singularity is split. This is attributed to using two SPs to create the mode $R_{1}^{2}$ (this does not happen in $\ell_{1}=2$ from $R_{1}^{1}$, figure $4 \mathrm{c}$, that is created with a single SP). Thus, the global OAM is 3 but, as we will show in figure $5 \mathrm{a}$, the singularity is also split in the focus intensity, to which it may contribute the instability of higher modes as well [36].
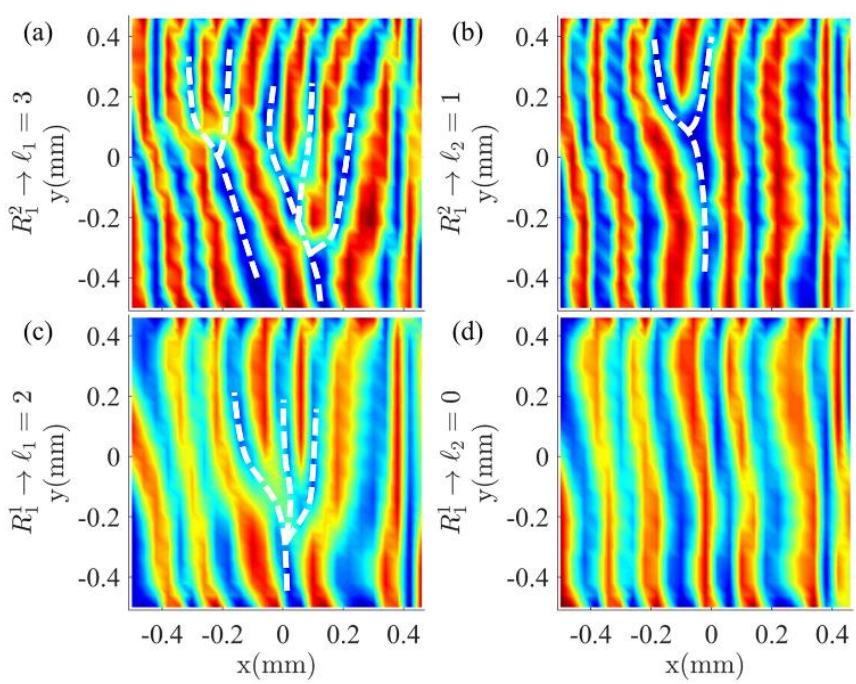

Figure 4. Experimental measurement of the OAM of the collimated vortex produced from $R_{1}^{2}(\mathrm{a}, \mathrm{b})$ and $R_{1}^{1}(\mathrm{c}, \mathrm{d})$. For $R_{1}^{2}$, the horizontal and vertical components of the beam have $\mathrm{OAM}=3$ (a) and $\mathrm{OAM}=1$ (b), respectively. For $R_{1}^{1}$, the horizontal and vertical components of the beam have $\mathrm{OAM}=2$ (c) and $\mathrm{OAM}=0$ (d), respectively. Dotted white lines serve as a guide for the reader to identify the dislocations in the fringes pattern. These features are the footprint of a spatial singularity, and their number reflects the topological charge. As can be observed, in (a) there are three dislocations, while in (b) there is only one, in (c) there are two and in (d) there are not dislocations, corresponding to the expected OAMs.

Then, we focused the ultrashort pulsed beam with the three different modes $R_{m}^{\ell_{0}}$ described before, and we measured its spatiotemporal profile (figure 5). Due to spatial inhomogeneity of the laser system (see figure 1, inset), we selected the central part of the beam (4.5-mm diameter). As predicted, the focused profile will have a ring with increasing diameter for higher OAMs (as seen in the numerical simulations in figure 3 ), while $\ell=0$ lacks any ring. Since the energy of each projection ( $\mathrm{X}$ and $\mathrm{Y}$ ) is the same, the peak intensity will be smaller for higher OAMs (the energy is spatially spread). In figure 5 (row 1), we show the profile at the focus for the input mode $R_{1}^{2}$ decomposed in OAM values 3 and 1 ( $\mathrm{X}$ and $\mathrm{Y}$, respectively). The different diameter of the ring is observed in the profiles, while in the total intensity (sum of $\mathrm{X}$ and $\mathrm{Y}$ projections), it is checked the prediction of 
higher peak intensity for the lower OAM (notice that the individual plots are normalized). In the second case, figure 5 (row 2), the radial mode $R_{1}^{1}$ is used to produce $\ell=2$ and $\ell=0$ (in $\mathrm{X}$ and $\mathrm{Y}$ ), thus the ring diameter in $\mathrm{X}$ is between the sizes found for $\ell=1$ and $\ell=3$, and the $Y$ projection is the diffraction limit of the input beam (being more intense in $\mathrm{Y}$ than $\mathrm{X}$ ). In the last case, radial polarization $R_{1}^{0}$ is decomposed in two vortices with opposite topological charge, $\ell= \pm 1$, having similar peak intensity. The transverse scan was done in a grid of $25 \times 25$ points using a step of $32 \mu \mathrm{m}$.

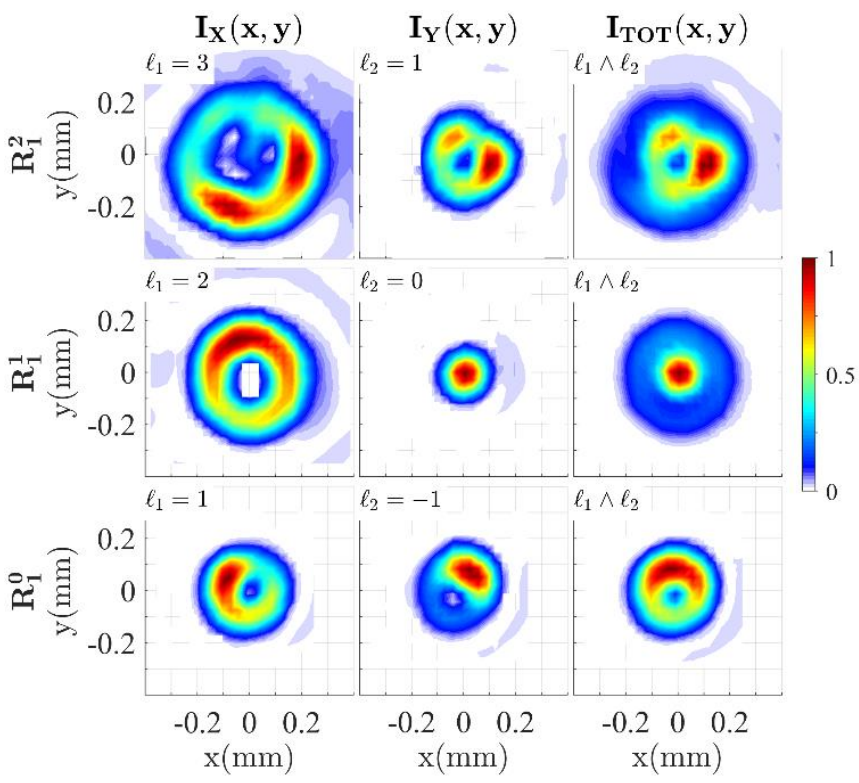

Figure 5. Spatial intensity profile of the focused beams. Columns 1, 2 and 3: X, Y and total components of the spatial intensity (OAM values inset). Rows 1, 2 and 3 (from the top) correspond to input $R_{1}^{2}, R_{1}^{1}$ and $R_{1}^{0}$, respectively. All intensity plots are individually normalized. In the animation of Supplementary Movie 1 the spatial profiles for different wavelengths are shown.

We further confirmed the OAM predicted by measuring the relative $\mathrm{X}$ and $\mathrm{Y}$ wavefronts, obtaining $\Delta \ell=2$ (due to $m=1$ ) in the three configurations. As we experimentally worked with ultrashort laser pulses, and we measured the temporallyand frequency-resolved spatial amplitude and phase of the collimated and focused vortices, we were able to check that the obtained results did not show significant differences as a function of the wavelength within the spectral bandwidth of the input beam, being constant in frequency as well as in time (see the animation in Supplementary Movie 1, corresponding to figure 5 , where it is shown that the spatial profiles are wavelength independent). The measured experimental intensity distributions in figure 5 were systematically observed. The deviations with respect to the simulated modes may be attributed to the spatial inhomogeneity of the input beam (CPA laser system) and to the misalignment of the Splates. At this respect, the inhomogeneity of the input beam profile (figure 1, inset) can affect the resulting profile after the beam manipulation. The alignment of the S-plates was carried out systematically, also checking the effect of the relative alignment in the case of using both S-plates simultaneously (to create the mode $R_{1}^{2}$ ), always finding the spatial distributions shown in figure 5 . Therefore, we attribute the deviation between simulations and experiments mainly to the nonuniformity of the input beam.

\section{Conclusion}

In conclusion, we demonstrate how spin-orbit manipulation, mediated by the polarization azimuthal index when using radially polarized beams, can be used to produce optical vortices with different topological charges. The proposal can be done in-line, without interferometric schemes. In contrast to previous works, the process is linear, thus all the energy is transferred to the produced vortices. It does not require tight focusing to mediate the angular momentum interaction (while being preserved under loose focusing), and constitutes an easy way to efficiently produce collinear vortices with different topological charges and with perpendicular linear polarization, so that they can be independently manipulated (e.g. balancing their relative intensity in pump-probe experiments) or used to simultaneously interact in the applications, for example in light-matter interaction, telecommunications, microprocessing, particle manipulation or attosecond pulse generation. The scheme can also be used to quantify the input OAM.

The process is valid for radial, azimuthal or intermediate spatially varying polarizations, being the polarization azimuthal index the key parameter for the vortices production. We have applied this method to ultrashort laser pulses, while it also operates for monochromatic plane waves, being either collimated or focused. Despite radially (or azimuthally) polarized beams not possessing spin angular momentum -due to being locally linearly polarized with different orientations--, we show that they can mediate to produce different orbital angular momenta through their polarization azimuthal index. Tuning the polarization azimuthal index can produce, for example, higher-order or fractional vortices.

\section{Acknowledgements}

The authors declare no conflicts of interest. This work was partially funded by Junta de Castilla y León (SA287P18) and FEDER Funds; Spanish Ministerio de Economía y 
Competitividad (MINECO) (FIS2017-87970-R, EQC2018004117-P); European Research Council (ENIGMA).

\section{References}

[1] Shen Y, Wang X, Xie Z, Min C, Fu X, Liu Q, Gong M and Yuan X 2019 Optical vortices 30 years on: OAM manipulation from topological charge to multiple singularities Light: Science \& Applications 8 1-29

[2] Dennis M R, O’Holleran K and Padgett M J 2009 Chapter 5 Singular Optics: Optical Vortices and Polarization Singularities Progress in Optics vol 53, ed E Wolf (Elsevier) pp 293-363

[3] Rego L, Román J S, Picón A, Plaja L and HernándezGarcía C 2016 Nonperturbative twist in the generation of extreme-ultraviolet vortex beams Physical Review Letters 117163202

[4] Meier M, Romano V and Feurer T 2007 Material processing with pulsed radially and azimuthally polarized laser radiation Applied Physics A 86 329-34

[5] Guclu C, Veysi M and Capolino F 2016 Photoinduced Magnetic Nanoprobe Excited by an Azimuthally Polarized Vector Beam ACS Photonics 3 2049-58

[6] Dorney K M, Rego L, Brooks N J, San Román J, Liao C T, Ellis J L, Zusin D, Gentry C, Nguyen Q L, Shaw J M, Picón A, Plaja L, Kapteyn H C, Murnane M M and Hernández-García C 2019 Controlling the polarization and vortex charge of attosecond high-harmonic beams via simultaneous spin-orbit momentum conservation Nature Photonics 13 123-30

[7] Berestetskii, V B, Pitaevskii, L P, Lifshitz, E M, 2012 Quantum Electrodinamics Volume 4, Oxford, Butterworth-Heinemann.

[8] Bliokh K Y, Rodríguez-Fortuño F J, Nori F and Zayats A v 2015 Spin-orbit interactions of light Nature Photonics 9 796-808

[9] Petersen J, Volz J and Rauschenbeutel A 2014 Chiral nanophotonic waveguide interface based on spin-orbit interaction of light Science 346 67-71

[10] Vázquez-Lozano J E and Martínez A 2018 Classical emergence of intrinsic spin-orbit interaction of light at the nanoscale Physical Review A 9733804

[11] Zhao L 2020 All-Optical Spin-Orbit Coupling of Light in Coherent Media Using Rotating Image Annalen der Physik 5321900371
[12] Ren H, Lin Y-H and Wu S-T 2006 Linear to axial or radial polarization conversion using a liquid crystal gel Applied Physics Letters 8951114

[13] Bashkansky M, Park D and Fatemi F K 2010 Azimuthally and radially polarized light with a nematic SLM Optics Express 18 212-7

[14] Wang X-L, Ding J, Ni W-J, Guo C-S and Wang H-T 2007 Generation of arbitrary vector beams with a spatial light modulator and a common path interferometric arrangement Optics Letters 32 3549-51

[15] Gecevičius M, Drevinskas R, Beresna M and Kazansky P G 2014 Single beam optical vortex tweezers with tunable orbital angular momentum Applied Physics Letters 104231110

[16] Beresna M, Gecevičius M, Kazansky P G and Gertus T 2011 Radially polarized optical vortex converter created by femtosecond laser nanostructuring of glass Appl. Phys. Lett. 98201101

[17] Du L, Man Z, Zhang Y, Min C, Zhu S and Yuan X 2017 Manipulating orbital angular momentum of light with tailored in-plane polarization states Scientific Reports 741001

[18] Porfirev A P, Ustinov A v and Khonina S N 2016 Polarization conversion when focusing cylindrically polarized vortex beams Scientific Reports 66

[19] Gori F 2001 Polarization basis for vortex beams Journal of the Optical Society of America A 18 1612-7

[20] Buono W T, Santiago J, Pereira L J, Tasca D S, Dechoum K and Khoury A Z 2018 Polarizationcontrolled orbital angular momentum switching in nonlinear wave mixing Optics Letters 43 1439-42

[21] Dai X, Li Y, Liu L, Liu K and Zheng M 2018 Determining the Charge of Vortex Beams by Focused Intensity After Polarization Modulation IEEE Photonics Technology Letters 30 487-90

[22] D’Errico A, Maffei M, Piccirillo B, de Lisio C, Cardano F and Marrucci L 2017 Topological features of vector vortex beams perturbed with uniformly polarized light Scientific Reports 740195

[23] Moreno I, Davis J A, Ruiz I and Cottrell D M 2010 Decomposition of radially and azimuthally polarized beams using a circular-polarization and vortex-sensing diffraction grating Optics Express 18 7173-83

[24] Xie Z, He Y, Chen X, Liu J, Zhou X, Ye H, Li Y, Chen S, Zhang X and Fan D 2019 Spatial phase and polarization retrieval of arbitrary circular symmetry 
singular light beams using orthogonal polarization separation Optics Express 27 27282-94

[25] Damzen M J, Kerridge-Johns W R and Geberbauer J W T 2019 Vortex mode transformation interferometry Journal of Optics 2215604

[26] Allegre O J, Perrie W, Edwardson S P, Dearden G and Watkins K G 2012 Laser microprocessing of steel with radially and azimuthally polarized femtosecond vortex pulses Journal of Optics 1485601

[27] Yang L, Chen X, Wang L, Hu Z, Xin C, Hippler M, Zhu W, Hu Y, Li J, Wang Y, Zhang L, Wu D and Chu J 2019 Targeted Single-Cell Therapeutics with Magnetic Tubular Micromotor by One-Step Exposure of Structured Femtosecond Optical Vortices Advanced Functional Materials 291905745

[28] Ni J, Wang C, Zhang C, Hu Y, Yang L, Lao Z, Xu B, Li J, Wu D and Chu J 2017 Three-dimensional chiral microstructures fabricated by structured optical vortices in isotropic material Light: Science \& Applications 6 e17011-e17011

[29] Ni J, Wang Z, Li Z, Lao Z, Hu Y, Ji S, Xu B, Zhang C, Li J, Wu D and Chu J 2017 Multifurcate Assembly of Slanted Micropillars Fabricated by Superposition of Optical Vortices and Application in High-Efficiency Trapping Microparticles Advanced Functional Materials 271701939

[30] JJ Nivas J, He S, Song Z, Rubano A, Vecchione A, Paparo D, Marrucci L, Bruzzese R and Amoruso S 2017 Femtosecond laser surface structuring of silicon with Gaussian and optical vortex beams Applied Surface Science 418 565-71

[31] Alonso B, Borrego-Varillas R, Mendoza-Yero O, Sola Í J, San Román J, Mínguez-Vega G and Roso L 2012 Frequency resolved wavefront retrieval and dynamics of diffractive focused ultrashort pulses Journal of the Optical Society of America B 29 1993-2000

[32] Alonso B, Pérez-Vizcaíno J, Mínguez-Vega G and Sola Í J 2018 Tailoring the spatio-temporal distribution of diffractive focused ultrashort pulses through pulse shaping Optics Express 26 10762-72

[33] Alonso B, Sola I J, Varela O, Hernandez-Toro J, Mendez C, San Roman J, Zair A and Roso L 2010 Spatiotemporal amplitude-and-phase reconstruction by Fourier-transform of interference spectra of highcomplex-beams Journal of the Optical Society of America B-Optical Physics 27 933-40
[34] Alonso B and Sola Í 2019 Measurement of Ultrashort Vector Pulses From Polarization Gates by In-Line, Single-Channel Spectral Interferometry IEEE Journal of Selected Topics in Quantum Electronics 258900307

[35] Alonso B, Lopez-Quintas I, Holgado W, Kazansky P G, Hernández-García C and Sola Í J 2019 Complete spatiotemporal and polarization characterization of ultrafast vector beams, submitted to Communications Physics, http://arxiv.org/abs/1912.11014

[36] Ricci F, Löffler W and van Exter M P 2012 Instability of higher-order optical vortices analyzed with a multipinhole interferometer Opt. Express 20 22961-75 